\title{
Ultrastructural Localization of Molecular Subtypes of Immunoreactive Neural Cell Adhesion Molecule (NCAM) in the Adult Rodent Striatum
}

\author{
Marian Difiglia,' Patricia Marshall,' Jonathan Covault, ${ }^{2}$ and Myuki Yamamoto ${ }^{3}$ \\ 'Department of Neurology, Massachusetts General Hospital and Harvard Medical School, Boston, Massachusetts 02114, \\ 2Department of Physiology and Neurobiology, University of Connecticut, Storrs, Connecticut 06268, and ${ }^{3}$ Department of \\ Biochemistry, Eunice Kennedy Shriver Center, Waltham, Massachusetts 02254
}

\begin{abstract}
Neural cell adhesion molecule (NCAM) is a plasma membrane glycoprotein that is thought to mediate adhesion between neuronal elements and play an important role in neural development. Although NCAM has been found in the adult brain, as well as the developing brain, little is known about its function or ultrastructural distribution. A monoclonal antibody which was directed against embryonic (E15-E17) mouse brain and identified 180 and $140 \mathrm{kDa}$ molecular weight forms of NCAM was used to examine the immunohistochemical localization of NCAM in the rodent striatum. Light microscopic results in the adult mouse and rat showed that most NCAM 100,140 immunoreactivity was localized to the relatively small population of medium and large-sized aspiny interneurons of the caudate nucleus and to the majority of neurons in the globus pallidus. Ultrastructural analysis revealed that reaction product in aspiny somata was present in discrete, closely spaced patches of cytoplasm along the inner face of the plasma membrane and was also prominent in somatic protrusions which were frequently apposed to synapsing axons. Distal aspiny and pallidal dendrites containing NCAM N00, 140 received numerous synaptic inputs. Within caudate neuropil NCAM ${ }_{180.140}$ was also present in spines with thin necks and small spine heads which were postsynaptic to unlabeled axon terminals and in preterminal (unmyelinated) axons and terminal boutons that issued from myelinated bundles and formed asymmetric synapses with unlabeled dendritic spines.

This study provides the first evidence in adult brain that NCAM $_{180,140}$ varies in extent and location within neurons. The heterogeneous distribution of NCAM 180,140 in neurons of the basal ganglia may be related to a number of functions such as maintaining or modulating the density and distribution of synaptic inputs and the formation of new contacts on dendritic spines.
\end{abstract}

Neural cell adhesion molecule (NCAM) is an integral membrane glycoprotein which mediates adhesion of neurons to neu-

\footnotetext{
Received Sept. 6, 1988; revised May 9, 1989; accepted May 12, 1989.

We are grateful to Dr. Andre Goffinet for giving us the AGl monoclonal antibody used in this study. This work was supported by NIH grants NS 16367 to M. DiFiglia and NS 24726 to M. Yamamoto.

Correspondence should be addressed to Dr. Marian DiFiglia, Laboratory of Cellular Neurobiology, Massachusetts General Hospital, MGH-East, Bldg. 149, East 13 Street, Charlestown, MA 02129.

Copyright $\odot 1989$ Society for Neuroscience $0270-6474 / 89 / 124158-11 \$ 02.00 / 0$
}

rons and to other types of cells (glia, muscle) (Edelman, 1983; Keilhauer et al., 1985). NCAM is highly concentrated in developing neural tissues (Beasley and Stallcup, 1987), and in vitro studies show that it is localized to axonal growth cones and filopodia (van den Pol et al., 1986) and may play a role in facilitating cell differentiation, aggregation, and migration, fiber bundle formation (Thiery et al., 1982; Daniloff et al., 1986; Beasley and Stallcup, 1987), and axon guidance (Silver and Rutishauser, 1984; Thanos et al., 1984; Tosney et al., 1986; see review by Covault, 1989). In the adult nervous system, NCAM is present in lower-molecular-wcight forms (MW $=180,140$, 120) that have fewer sialic acid residues and a greater homophilic binding than in the embryo (MW 200-230). Although NCAM in most regions of the adult brain is substantially lower in content than during development (Daniloff et al., 1986), it has been localized by immunofluorescence to some mature neurons (cerebellum, retina; Beasley and Stallcup, 1987), where its function is unknown. Given the continually increasing evidence for neuronal plasticity in the adult brain, it is possible that NCAM functions to some extent in mature neurons to mediate the cell-cell contacts required for neuronal regeneration and synapse formation. Studies in the peripheral nervous system suggest that NCAM might be involved in recovery after injury. Following denervation, NCAM was significantly elevated above its normal low levels in adult rat skeletal muscle and in the axon terminals reinnervating the muscle (Covault and Sanes, 1985; Reiger et al., 1985).

In this study we examined the electron microscopic localization of immunoreactive NCAM in the basal ganglia which have been the focus of research interest in one of our laboratories for many years.

\section{Materials and Methods \\ Production of monoclonal antibody AGI}

$\mathrm{BALB} / \mathrm{c}$ mice were injected intraperitoneally 3 or 4 times at 2 week intervals with a homogenate prepared from the brains of rat embryos (E15-E17) that was mixed with polyinosilic and polycytidilic acid (50 $\mu \mathrm{g}$ of adjuvant per injection). Three to four days following the final injection, the mice were sacrificed and their spleens removed. Spleen cells were dissociated mechanically and fused with NS-1 myeloma cells by the method of Kohler and Milstein (1976). Hybridoma cells were then selected by culturing in the presence of hypoxanthine-animopterine-thymidine (HAT).

The AGl antibody was identified in supernatant derived from a clone of hybridoma cells in the course of immunohistochemical screening against tissue sections obtained from the forebrain of embryonic mice. The antibody was found to be of the IgG subclass. 
Antigen identification with the AG1 monoclonal antibody

Two separate experiments were performed.

Experiment $A$. The brains from embryonic and adult mice $(\mathrm{C} 3 \mathrm{H} \times$ C57) were homogenized at $0^{\circ} \mathrm{C}$ in $50 \mathrm{~mm}$ Tris buffer containing $5 \mathrm{~mm}$ EGTA, $1 \mathrm{~mm}$ (PMSF), $25 \mu \mathrm{M}$ leupeptin, $0.1 \%$ aprotinin to inhibit proteases. After centrifugation at $5000 \mathrm{rpm}$, the supernatant was separated by $7.5 \% \mathrm{SDS} /$ polyacrylamide gels either with or without protease inhibitors. Polypeptides were then transferred from the gel to nitrocellulose paper ( $0.45 \mu \mathrm{m}$, Millipore) by the method of Towbin et al. (1979). The blots were then incubated for $30 \mathrm{~min}$ in PBS containing $10 \%$ normal goat serum (NGS), washed briefly with PBS containing $1 \%$ NGS (NGSPBS) and then either incubated overnight at $4^{\circ} \mathrm{C}$ or for $2-3 \mathrm{hr}$ at room temperature with supernatant from the AG1-producing hybridoma. After exposure to the primary antibody, the nitrocellulose paper was washed with NGS-PBS $(3 \times 10 \mathrm{~min})$, incubated for $2 \mathrm{hr}$ at room temperature with peroxidase-conjugated goat anti-mouse IgG (1:1000 in NGS-PBS), washed with PBS $(3 \times 10 \mathrm{~min})$, and reacted with $0.05 \% \mathrm{DAB}$ and $0.02 \%$ $\mathrm{H}_{2} \mathrm{O}_{2}$.

A neuraminidase reaction was also performed prior to gel electrophoresis by incubating $60 \mathrm{mg}$ of homogenized tissue with 0.5 units of neuraminidase (Vibrio cholerae; Cal-biochem) at $37^{\circ} \mathrm{C}$ and pH 5.5 overnight. As a control, homogenate was incubated with the same solution without neuraminidase. In one case after blotting, the nitrocellulose paper was incubated with neuraminidase solution in order to determine whether the sialic acid portion is an immunodeterminant of AGl antibody.

Experiment $B$. Affinity-purified rat brain NCAM and rabbit antiNCAM polyclonal antibody were prepared as previously described (Covault and Sanes, 1986). Samples of NCAM (160 ng) or Nonidet P-40 ( $0.5 \%$ in PBS plus $1 \mathrm{~mm}$ EDTA, $1 \mathrm{~mm}$ phenylmethylsulfonylchloride) extracts of rat or mouse brain $(3 \mu \mathrm{g})$ were electrophoresised in $7 \% \mathrm{SDS} /$ polyacrylamide gels, transferred to nitrocellulose filters $(0.45 \mu \mathrm{m}$, Bio$\mathrm{Rad})$, and reacted with primary (AGl and a polyclonal anti-NCAM) and secondary antibodies as previously described (Covault et al., 1986).

\section{Immunohistochemistry}

Eight adult animals $(n=3 \mathrm{C} 3 \mathrm{H} \times \mathrm{C} 57$ hybrid mice and $1 \mathrm{Balb} / \mathrm{c}$ mouse and 4 Sprague-Dawley rats were perfused through the heart, and the brains were removed immediately and sectioned or stored in phosphate buffer (PB) overnight before sectioning. A variety of fixative preparations were tried which gave satisfactory results in the mouse $(2-4 \%$ paraformaldehyde in phosphate buffer or periodate-lysine-paraformaldehyde), but in the rat staining and tissue preservation compatible with ultrastructural study were achieved by perfusing with $2 \%$ paraformaldehyde in PB followed by a brief incubation of the brain in the same fixative to which $0.1 \%$ glutaraldehyde had been added.

Vibratome sections through the basal ganglia, $30-40 \mu \mathrm{m}$ thick, were incubated in $1 \%$ normal goat serum, washed in PB and incubated in the NCAM monoclonal antibody (AG1) at dilutions of $1: 10$ or $1: 20$ for 1-2 d. Detergents were not used to increase penetration since it had been determined that the addition of $0.01 \%$ saponin to the anti-NCAM incubation solution resulted in markedly diminished membrane staining. Control sections were treated identically except that the AG1 monoclonal antibody was omitted; under these conditions no specific staining was present. Sections were washed in PB and incubated for $90 \mathrm{~min}$ in a biotin-conjugated horse anti-mouse IgG (1 drop/15 ml PB) and then with avidin-peroxidase complex (Vectastain, Vector Laboratories, Burlingame, CA). The sections were washed in $\mathrm{PB}$ and treated for 6-10 min in $0.01 \%$ diaminobenzidine and $0.001 \% \mathrm{H}_{2} \mathrm{O}_{2}$. Sections were treated with $1 \%$ osmium tetroxide, stained en bloc with $1 \%$ uranyl acetate, and embedded flat in Epon-Araldite. Thin sections were mounted on Formvar-coated slot grids and examined in a Jeol $100 \mathrm{CX}$ electron microscope.

Figure 1. a, AG1 monoclonal antibody reacts with rat brain homogenates. Western blots of adult (lanes 1-3) and E15 (lanes 4-6) brain homogenate reacted with AG1 antibody. Lanes 3 and 6, no treatment; lanes 2 and 5, homogenate was incubated with buffer (pH 5.5) prior to gel electrophoresis; lanes 1 and 4, homogenate was incubated with neuraminidase in buffer prior to gel electrophoresis. Arrowheads at right identify molecular weights of 230,180 , and $140 \mathrm{kDa}$ from top to bottom, respectively. Note that the AG1 antibody identifies a 140 kDa NCAM in the adult (lane 3) and not in the embryonic tissue (lane 6) and that

\section{Adult E15}
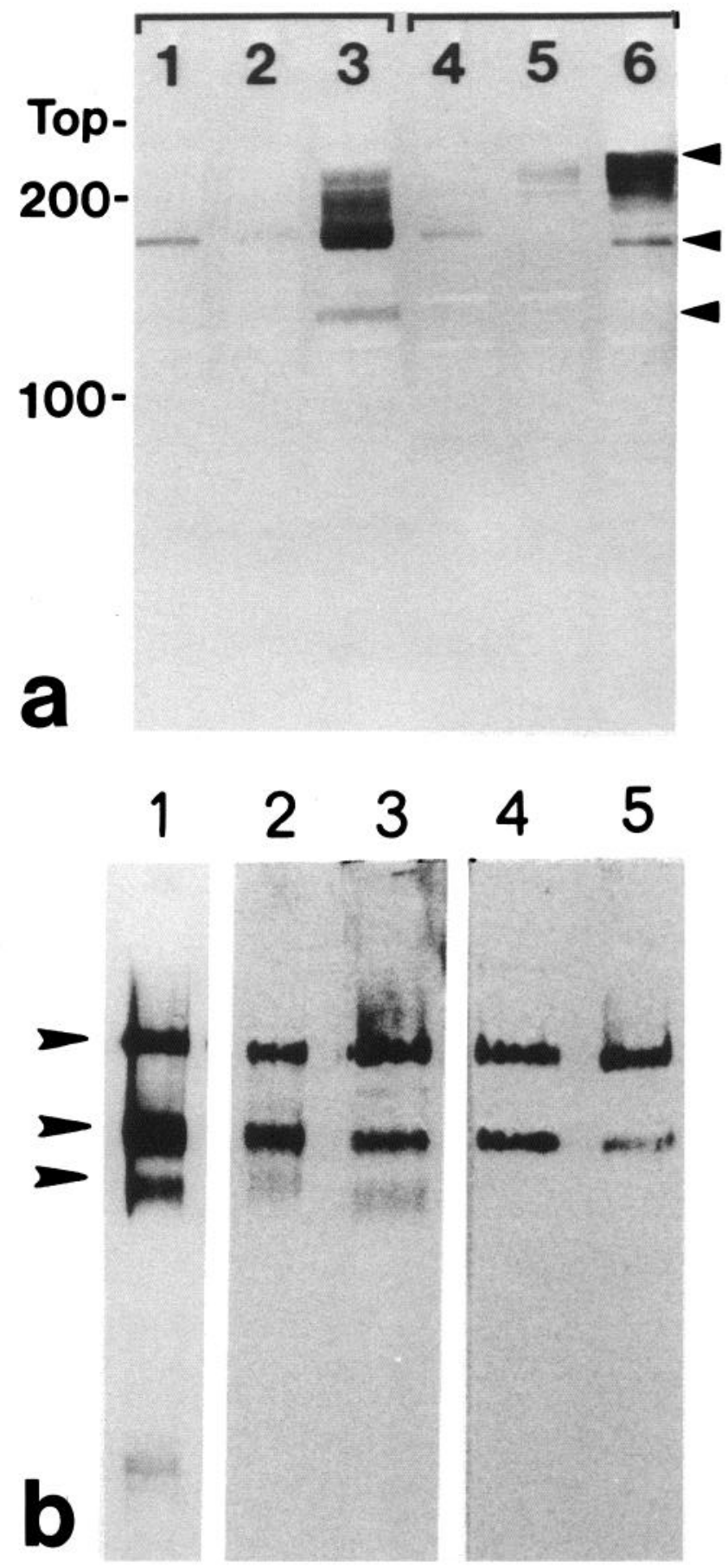

the $180 \mathrm{kDa}$ species is more prominent in the adult than in the embryo. $b$, AG1 monoclonal antibody recognizes the 180 and $140 \mathrm{kDa}$ forms of rat NCAM. Lane 1 shows a silver-stained SDS gel containing 200 ng of affinity-purified NCAM from adult rat brain. Arrowheads indicate the positions of the 3 principle forms of NCAM: 180,140, and 120 $\mathrm{kDa}$ (from top to bottom). Immunoblots of affinity-purified adult brain NCAM, $160 \mathrm{ng}$ (lanes 2 and 4) and detergent extracts of adult brain, 3 $\mu \mathrm{g}$ (lanes 3 and 5 ) probed with either a polyclonal anti-NCAM antibody (lanes 2 and 3) or with AG1 monoclonal antibody (lanes 4 and 5) and appropriate peroxidase-conjugated second antibodies. AG1 reacts with the 180 and $140 \mathrm{kDa}$ forms of rat NCAM but not the smaller $120 \mathrm{kDa}$ form (position identified by lowest arrowhead) as compared with the polyclonal anti-NCAM antibody, which reacts with all 3 molecularweight species. 

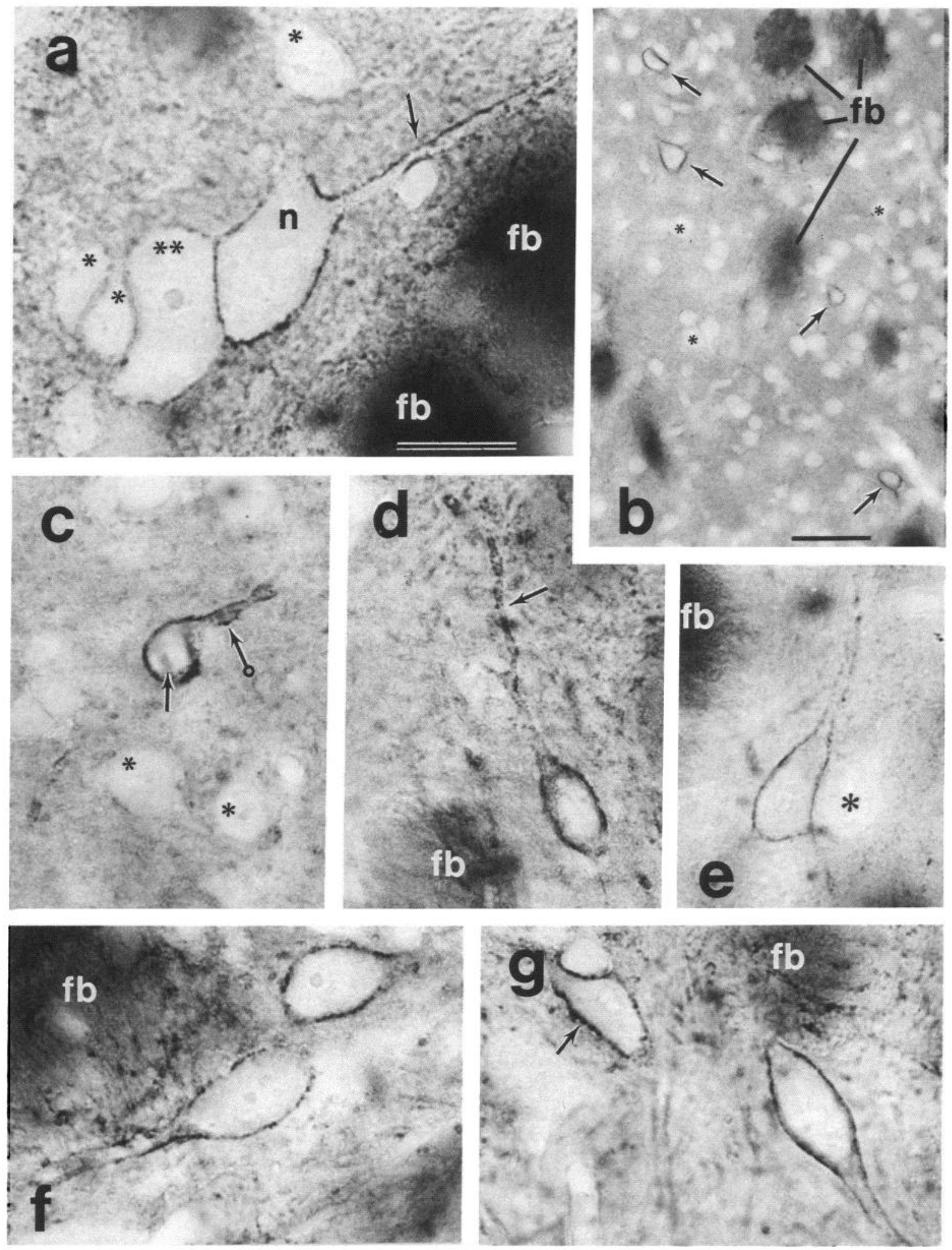

Figure 2. Osmium-treated and Epon-embedded vibratome sections show immunoreactive NCAM in the rat $(a, c-d)$ and mouse $(b, e)$ caudate nucleus and rat globus pallidus $(f, g)$. Fiber bundles, $(f b)$ appear black due to postfixation with osmium tetroxide. In the caudate nucleus, peroxidase reaction product outlines a large neuron $(n)(a)$ and medium-sized cells $(b-e)$. In $b$, neurons that are NCAM-positive (arrows) represent a small portion of the total population. Asterisks identify some unlabeled neurons. In $c$, the indented nucleus (arrow) and varicose dendrite (ringed arrow) identify the medium-sized neuron as an aspiny type. Primary dendrites (arrow in $c$ ) and secondary dendrites (arrow in $d$ ) are also labeled. Medium (asterisk in $a-e$ ) and large neurons (double asterisks in $a$ ) not displaying NCAM immunoreactivity are also visible. Beaded appearance of the reaction product is apparent in many locations in the NCAM-positive caudate neurons and also in the pallidal neurons (note beading at arrow in $g$ ). Scale bar in $a$ is $20 \mu \mathrm{m}$ and also applies to $c-g$; scale bar in $b$ is $100 \mu \mathrm{m}$. 


\section{Results}

Molecular subtypes of $N C A M(180,140, k D a)$ are recognized by the AG1 monoclonal antibody

On Western blots, the most intense reaction of AG1 to brain homogenates was seen as a broad band in the molecular-weight range of 180 to about $230 \mathrm{kDa}$ (Fig. 1 a). The band with highermolecular-weight forms (200-230 kDa) was more prominent in the embryonic brain, whercas the band with lower-molecularweight constituents $(180 \mathrm{kDa})$ was more prominent in the adult brain. In the adult, but not the embryonic brain, immunoreactive bands were also observed at $140 \mathrm{kDa}$.

Brief treatment with neuraminidase reduced the broad band (180-230 kDa) derived from both adult and embryo to a single $180 \mathrm{kDa}$ band (Fig. $1 a$, lanes 1 and 4), indicating that variations in sialic acid residues account for the broader spectrum of molecular weights. Neuraminidase treatment did not affect the 140 $\mathrm{kDa}$ band from the adult brain. Also, neuraminidase treatment of the blotted nitrocellulose paper did not abolish immunoreactivity, indicating that the epitope recognized by the AG1 monoclonal antibody does not include sialic acid residues. This electrophoretic profile and apparent decrease in sialic acid content of the AG1 antigen in adult versus embryonic brain suggested that the AG1 monoclonal antibody recognizes an epitope on NCAM.

To further identify the specificity of AG1 to the NCAM molecule, we compared the specificity of the AG1 antibody and a previously characterized polyclonal anti-NCAM antibody (Fig. $1 b$, lane 1). Results (Fig. 1b, lanes 4 and 5) showed that AG1 reacted with the 180 and $140 \mathrm{kDa}$ and not the $120 \mathrm{kDa}$ forms of rat NCAM, unlike the polyclonal anti-NCAM, which reacted with all 3 molecular-weight species (Fig. $1 b$, lanes 2 and 3). AG1 also recognized the 180 and $140 \mathrm{kDa}$ but not the $120 \mathrm{kDa}$ forms of mouse brain NCAM (data not shown). These results, coupled with the immunochemical localization of the AG1 epitope to the cytoplasmic side of the plasmalemma (see below), suggest that AGl binds to a cytoplasmic domain of rodent NCAM similar to that encoded by exons 16,17 , and 19 in chicken NCAM and common to both the 180 and $140 \mathrm{kDa}$ forms but absent from the $120 \mathrm{kDa}$ form, which lacks a transmembrane or cytoplasmic domain (Gennarini et al., 1984; Cunningham et al., 1987). The reactivity of AG1 is thus very similar to that previously described for the rat monoclonal P61 antibody (Gennarini et al., 1984).

\section{Immunocytochemical localization of NCAM ${ }_{180,140}$ in adult basal ganglia}

In both the rat (Fig. 2, $a, c, d$ ) and mouse (Fig. 2, $b, e$ ) immunoreactive NCAM was localized to medium $(10-18 \mu \mathrm{m}$; Fig. 2 , $b-e)$ and large (25-30 $\mu \mathrm{m}$; Fig. $2 a)$ sized neurons throughout the caudate nucleus. Peroxidase reaction product within somata was localized to peripheral regions of the cell and also outlined the primary (arrows in Fig. 2, a-e) and secondary dendrites (arrow, Fig. 2d). The NCAM 180,140 -positive, medium-sized neurons represented a small percentage of all caudate neurons (Fig. $2 b$ ); immunoreactive medium-sized cells had indented nuclei (arrow in Fig. 2c) and varicosities in some dendrites (ringed arrow in Fig. $2 c$ ); both features are characteristic of medium aspiny type neurons (DiFiglia et al., 1980). Peroxidase label appeared as a stippled deposit along the inside of the plasma membrane and adjacent cytoplasm in labeled cell bodies and dendrites.

In contrast to the caudate nucleus, nearly all of the neuronal somata and dendrites of the globus pallidus in both the rat (Fig. $2, f, g)$ and mouse exhibited immunoreactive NCAM $_{180,140}$. Like the labeled aspiny neurons in the caudate nucleus, reaction product in pallidal cells appeared beaded along some portions of the plasma membrane and adjacent cytoplasm (example at arrow in Fig. 2g).

$N C A M_{180,140}$ is localized in a patchy pattern along the inside of the plasma membrane and adjacent cytoplasm of caudate aspiny neurons and cells of the globus pallidus

In all animals examined at the ultrastructural level $(n=2$ rats and 2 mice), the same distribution of immunoreactive NCAM $_{180,140}$ was obscrved. Aspiny neurons (Fig. $3 a$ ) and dendrites (Fig. $3 b$ ) in the caudate nucleus exhibited irregularly spaced patches of peroxidase reaction product along the inside of the plasma membrane and within the cytoplasm that extended about $0.5 \mu \mathrm{m}$ from the plasmalemma. Of particular interest was the consistent presence of $\mathrm{NCAM}_{180,140}$ in somatic protrusions which were found in both medium (Fig. 3c) and large (Fig. 3d) aspiny neurons when followed in serial sections. The labeled protrusions were frequently postsynaptic (Fig. $3 c$ ) and extended into neuropil, where boutons and thin unmyelinated axons were present.

We noted that a high proportion of the profiles contiguous with $\mathrm{NCAM}_{180,140}$-labeled aspiny somata and primary dendrites

\begin{abstract}
Figure 3. Electron micrographs of immunoreactive NCAM in caudate aspiny neurons. $a$, The soma of a medium aspiny neuron with typical indented nucleus exhibits patches of NCAM reaction product within the cytoplasm near the plasma membrane (arrows). Region in boxed area is shown in $c$. Scale bar, $2 \mu \mathrm{m}$. $b$. Primary dendrite of an aspiny neuron exhibits patches of reaction product (arrows) in the cytoplasm and adjacent plasma membrane. Ringed arrow identifies small NCAM-positive dendrite. Scale bar, $1 \mu \mathrm{m}$. $c$, NCAM-positive somatic protrusion (taken from boxed region in $a$ ) is bordered by small cross-section profiles $\left(^{*}\right)$, which are probably unmyelinated axons, and an unlabeled axon terminal $(a x)$ that forms a contact at arrow. Scale bar, $0.5 \mu \mathrm{m}$. $d$, A somatic protrusion belonging to the soma of a large caudate aspiny neuron extends into neuropil that contains small axon fibers and terminals $\left(^{*}\right)$. $s$, spine. Scale bar, $0.5 \mu \mathrm{m}$.
\end{abstract}

Figure 4. NCAM-positive dendrites (den) in the caudate nucleus $(a)$ and globus pallidus $(b)$ of the same rat show a striking similarity in innervation by axon terminals $\left(^{*}\right)$. The NCAM profile in $a$ is a dendritic varicosity belonging to an aspiny neuron. Note also that the axon terminals contacting the caudate dendrite appear similar to those synapsing with the pallidal dendrite; in fact, the axons may all derive from neostriatal spiny neurons. Scale bar in $a$ is $0.5 \mu \mathrm{m}$ and also applies to $b$. $c$, Portion of an NCAM-immunoreactive cell in the globus pallidus. Immunoreactivity is present along the plasma membrane and adjacent cytoplasm on the left side of the cell, especially between the arrows. Scale bar, $1 \mu \mathrm{m} . d$, Large-diameter NCAM-positive dendrite, scctioned longitudinally in the rat globus pallidus, exhibits patches of reaction product that are distributed similarly to those seen in the dendrites of aspiny caudate neurons (see Fig. $3 b$ ). Scale bar, $1 \mu \mathrm{m}$. 


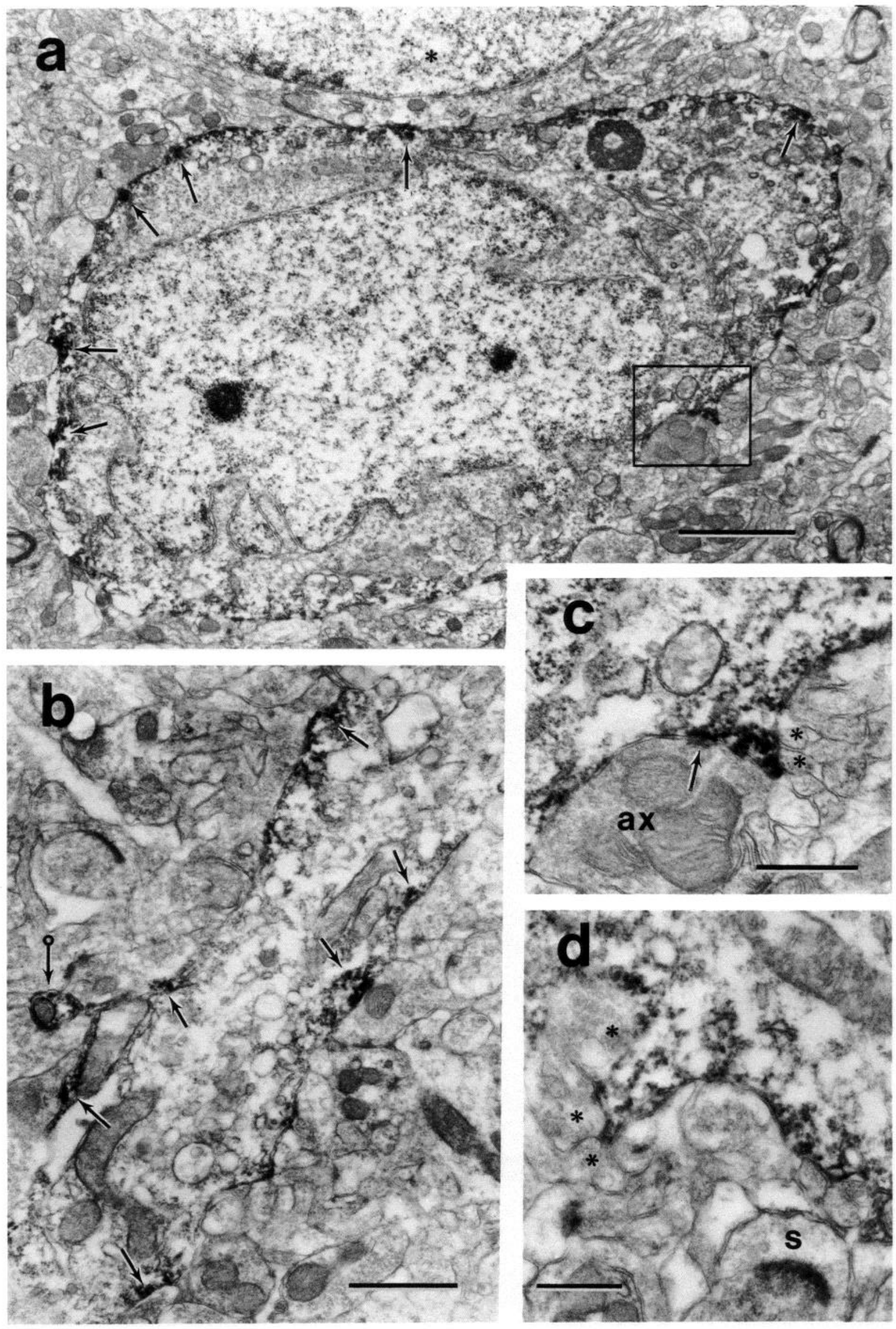



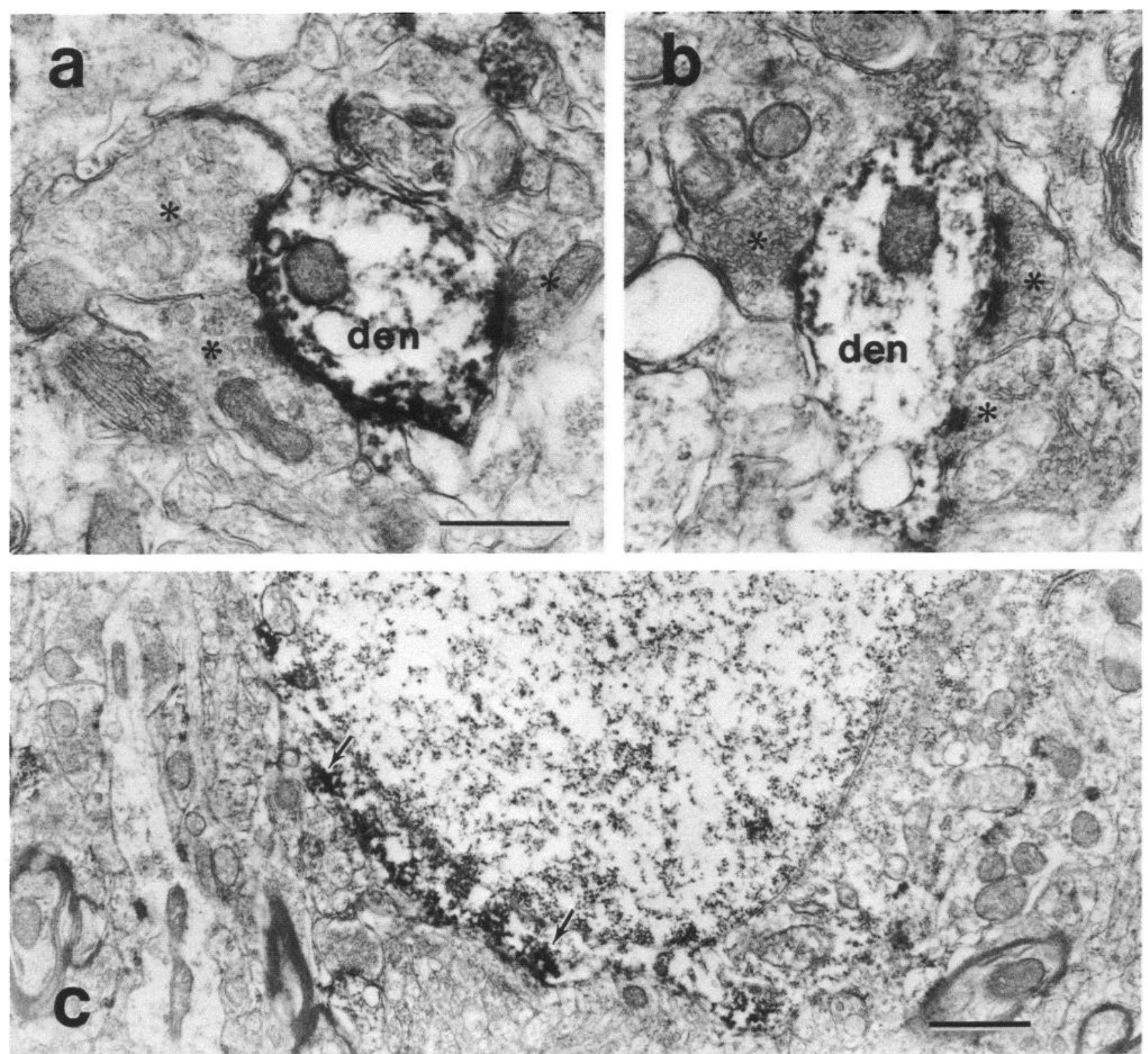

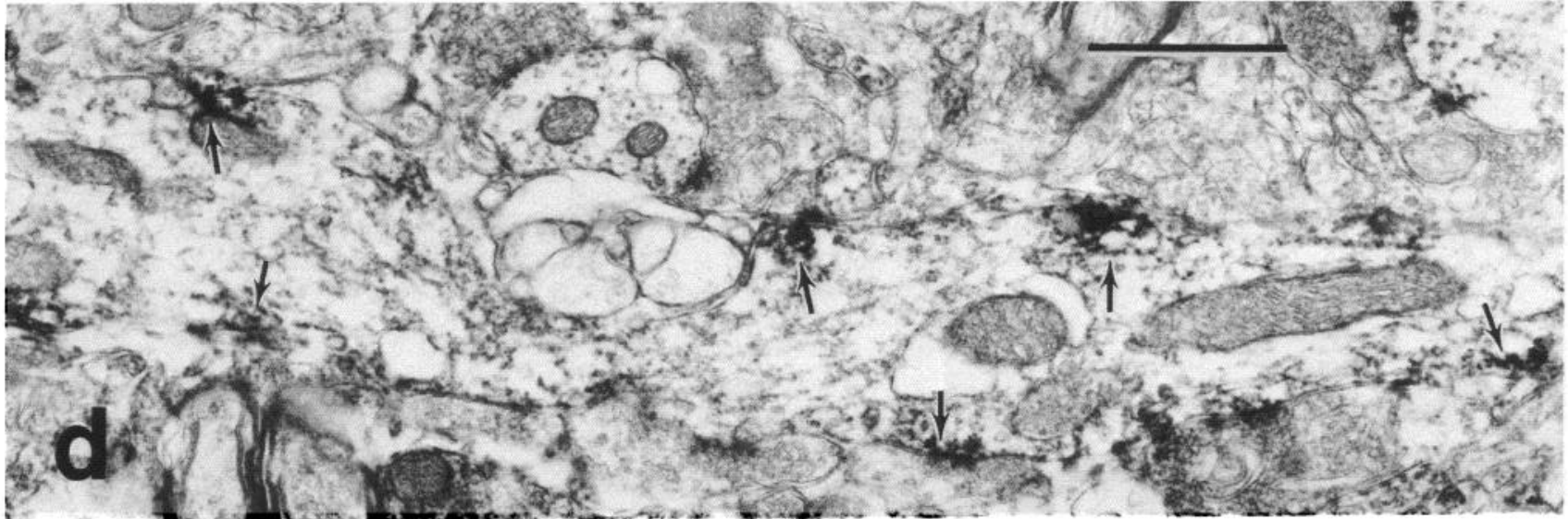



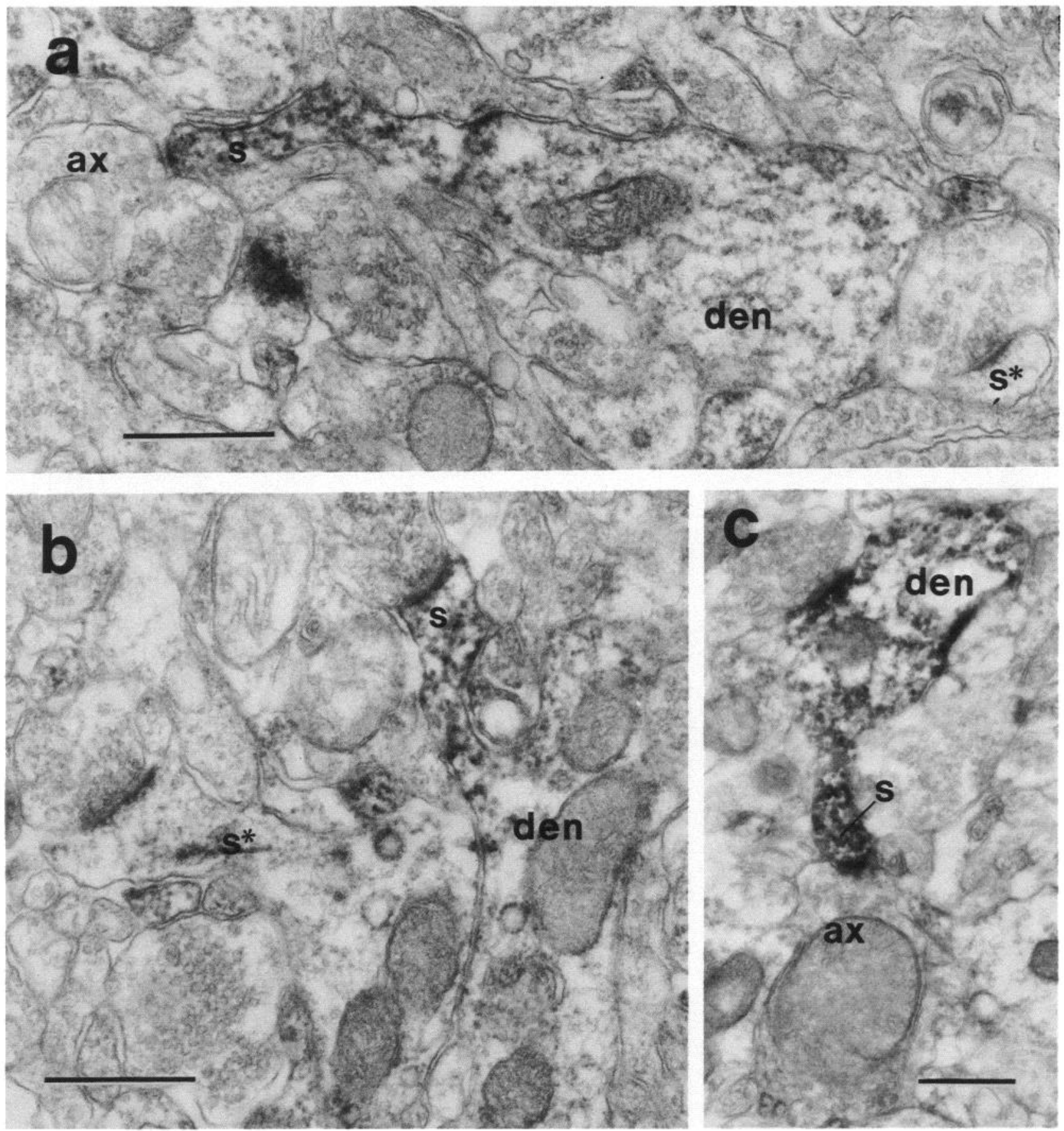

Figure 5. NCAM-immunoreactive spines in the adult rat caudate nucleus. $a$, A dendrite (den) shows relatively little immunoreactivity compared with the emerging long thin, spine $(s)$, which is filled with reaction product and forms a synapse with axon terminal ( $a x)$. $s^{*}$ is an unlabeled spine. Scale bar, $0.5 \mu \mathrm{m}$. $b$, A thin spine $(s)$ is NCAM positive and arises from a dendrite $(d e n)$ that contains little reaction product. $s^{*}$ is an unlabeled spine. Both $s$ and $s^{*}$ are postsynaptic. Scale bar, $0.5 \mu \mathrm{m}$. $c$, An infrequently observed example of an NCAM-positive dendrite (den) and its emerging spine $(s)$, both of which are heavily labeled with reaction product. $s$ is postsynaptic to axon terminal (ax). Scale bar, $0.5 \mu \mathrm{m}$.

were axons (Fig. 3, $a, b$ ), most of which in a single thin section did not form synaptic contacts with the labeled cell. Patches of immunoreactive $\mathrm{NCAM}_{180.140}$ along the plasma membranes of labeled aspiny neurons and primary dendrites frequently abutted a synapsing axon (Fig. 3, $a-c$ ), $\mathrm{NCAM}_{180,140}$-positive patches of membrane, however, were also found adjacent to axons not forming synaptic contacts, as well as other profiles which in- cluded somata, dendrites, and glial processes. Immunoreactive aspiny somata and dendrites were only occasionally seen in apposition to neuronal profiles that were also $\mathrm{NCAM}_{180,140}$ positive.

The distal dendrites of aspiny neurons exhibited patches of peroxidase reaction product along their plasma membranes and, unlike more proximal regions of aspiny neurons, the cytoplasm 
of small labeled dendrites had a more extensive deposition of reaction product. When viewed in cross section, small immunoreactive NCAM dendrites were surrounded by boutons and up to 3 terminals made synaptic contact within a single thin section (Fig. 4a).

The somata (Fig. 4c) and dendrites (Fig. 4, b, d) of pallidal neurons exhibited the same pattern of extensive but irregularly spaced patches of immunoreactive NCAM along their plasma membranes and adjacent cytoplasm as those of caudate aspiny neurons. NCAM-positive pallidal cells were surrounded by synapsing terminals, an ultrastructural feature highly characteristic of pallidal architecture (DiFiglia et al., 1980; DiFiglia and Rafols, 1988). A striking similarity was apparent in the heavy synaptic innervation of immunoreactive NCAM dendrites belonging to pallidal cells and some caudate aspiny neurons (compare caudate dendrite in Fig. $4 a$ with pallidal dendrite in Fig. 4b).

In the globus pallidus, as well as the neostriatum, glial elements were devoid of $\mathrm{NCAM}_{180.140}$ immunoreactivity.

\section{Thin spines of caudate spiny neurons are selective sites of NCAM ${ }_{180.140}$ immunoreactivity}

Unlike aspiny neurons, the somata and dendritic shafts of caudate spiny neurons were largely devoid of NCAM $_{180,140}$. A rarely found small NCAM $_{180,140}$-positive dendrite with emerging spine is shown in Figure $5 c$. Frequently, small spines with NCAM immunoreactivity were encountered in the caudate neuropil. The thin necks of labeled spines were $0.10-0.15$ in diameter and $0.40-1.0 \mu \mathrm{m}$ in length. The endings or heads of labeled spines were either equal (Fig. $5 a$ ) or somewhat larger in size than the spine neck (Fig. 5, $b, c$ ). Peroxidase reaction product was visible along the inside of the plasma membrane and also within the cytoplasm of the spine neck and head. The shafts of dendrites from which labeled spines arose were either unreactive or partly labeled near the base of the spine (Fig. 5, $a, b$ ). Some of the synaptic contacts onto NCAM-positive spines appeared "immature" with wide synaptic gaps and few vesicles accumulated at the presynaptic site (Fig. 5a). It is worth emphasizing that only thin spines with small heads contained $\mathrm{NCAM}_{180,140}$ immunoreactivity; large spines of various shapes were never labeled even when they were in close proximity to $\operatorname{NCAM}_{180,140^{-}}$ positive spines (Fig. $5 b$ ).

\section{Small axon terminals that form asymmetric synapses with dendritic spines are $N C A M_{180,140}-$ positive in adult caudate neuropil}

A minor homogeneous population of thin unmyelinated axons and small boutons were heavily labeled with peroxidase reaction product. NCAM-positive preterminal axons and boutons were frequently found in myelinated bundles (Fig. $6 a$ ). Small unlabeled dendritic spines entering the bundles were postsynaptic to NCAM-positive terminals. Similar NCAM-positive boutons were frequently found in regions of caudate neuropil containing clusters of small unmyelinated axons and spines (Fig. $6, b, c$ ). Labeled boutons were relatively small compared with unlabeled axon terminals, ranging from $0.25-0.5 \mu \mathrm{m}$ in size, and formed asymmetric synapses only with small spines. Two features of the NCAM-positive boutons suggested that they were of extrinsic origin: their presence in striatal fiber bundles and their asymmetric contacts, which are characteristic of most caudate afferent axons but not intrinsic axons.

\section{Discussion}

The role of NCAM in the adult brain is unknown, but various in vitro studies have suggested that membrane-membrane contacts mediated by NCAM permit a variety of cell interactions to occur such as the regulation of transmitter content and the formation of specialized junctions (see review by Rutishauser et al., 1988). Moreover, the degree of adhesion between cell membranes is greatly influenced by both the overall expression of NCAM and the molecule's content of polysialic acid (PSA); low PSA content, as found in the adult form of the molecule, promotes greater adhesion. As discussed further below, understanding the role of NCAM within different types of adult neurons may depend in part on the extent and location of NCAM, unique features of neuronal and synaptic architecture, and known functional properties of neurons.

\section{$N C A M_{180,140}$ is heterogeneously distributed in the adult basal ganglia}

NCAM localization has been examined in relatively few regions of the adult CNS (spinal cord, retina, tectum, and cerebellum), and mostly with immunofluorescent methods (Daniloff et al., 1986; Beasley and Stallcup, 1987). Findings have generally suggested that adult forms of NCAM are homogeneously and ubiquitously distributed with relatively little preference for different neuronal populations. One biochemical study indicates, however, that there are wide variations in concentrations of NCAM observed in different adult CNS nuclei (the basal ganglia were not examined) (Chuong and Edelman, 1984).

Our results in the basal ganglia provide the first morphological evidence using immunoperoxidase methods that adult NCAM may vary in its extent and location within different adult neuronal populations. Neurons of the globus pallidus exhibited $\mathrm{NCAM}_{180,140}$ immunoreactivity as extensively as neurons in other regions previously examined. However, in the caudate nucleus extensive NCAM labeling was confined to a relatively small proportion of the total caudate cell population (5-6\%), the medium and large aspiny neurons. $\operatorname{NCAM}_{180,140}$ immunoreactivity was not visible at the light microscopic level in the majority of neostriatal neurons, the medium spiny cells. However, ultrastructural study showed that $\mathrm{NCAM}_{180,140}$ was distributed selectively to some dendritic spines belonging to spiny cells.

\section{Features of aspiny caudate neurons and pallidal cells and the presence of $N C A M$}

In rodent, the medium and large caudate aspiny interneurons account for only about 5 and $1 \%$, respectively, of the total caudate cell population. Somatostatin and neuropeptide $Y$ are contained in the medium aspiny cell and $\mathrm{ACh}$ is the neurotransmitter of the large aspiny neurons (DiFiglia and Aronin, 1982; Phelps et al., 1985; DiFiglia, 1987). The cell biology of aspiny neurons has been of considerable interest since the recent observation that both types of aspiny interneurons are relatively spared in Huntington's disease, which results in severe atrophy of the caudate nuckeus and neurodegenerative changes in medium spiny cells (Ferrante et al., 1985; Graveland et al., 1985).

The similarly extensive membrane distribution of $\mathrm{NCAM}_{180,140}$ in medium and large caudate aspiny interneurons and in pallidal projection neurons, may be related to a common feature of these neurons, their high density and variety of synaptic contacts. 

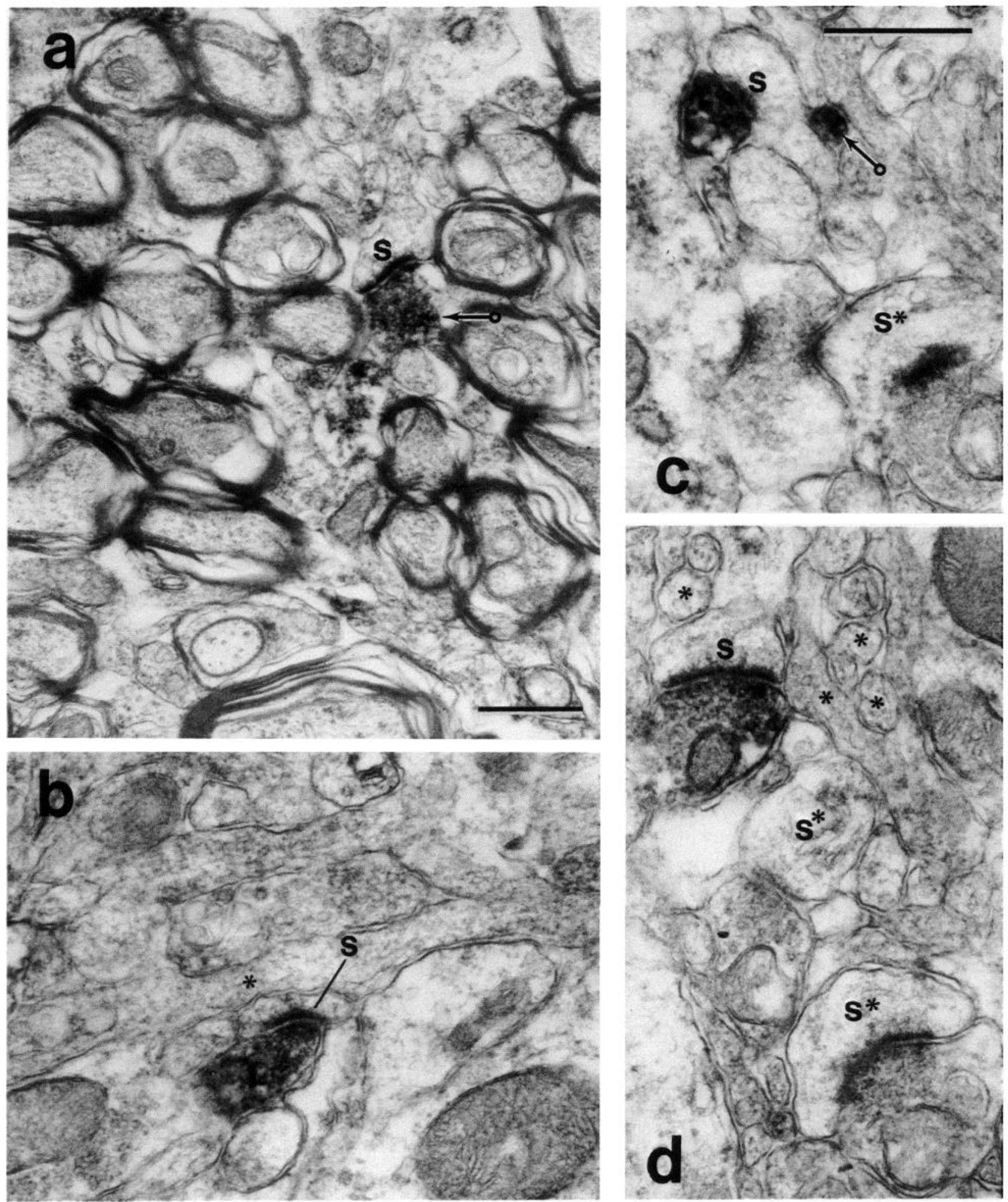

Figure 6. Immunoreactive NCAM axons in the adult rat caudate nucleus. $a$, An NCAM-positive terminal (ringed arrow) emerges from a preterminal axon that is also NCAM immunoreactive and forms a synapse with a spine $(s)$. Both the axon and spine are embedded within a striatal bundle composed of myelinated fibers. Scale bar, $0.5 \mu \mathrm{m}$. $b-d$, Examples of the immunoreactive NCAM terminals found in caudate neuropil. Note that all the immunoreactive terminals make asymmetric synapses with spines $(s)$ and that unlabeled postsynaptic spines $\left(s^{*}\right.$ in $c$ and $\left.d\right)$ and small unmyelinated fibers $\left({ }^{*}\right.$ in $b$ and $\left.d\right)$ contribute to the surrounding neuropil. Note also at the ringed arrow in $c$, the typically small size of an NCAMimmunoreactive fiber in the caudate nucleus. Scale bar in $c$ is $0.5 \mu \mathrm{m}$ and also applies to $b$ and $d$. 
Combined Golgi-EM studies have shown that the varicose portions of distal dendrites of medium and large aspiny neurons are highly innervated by synapsing axons (DiFiglia et al., 1980; DiFiglia and Carey, 1986), a feature which applies even more extensively to the surfaces of pallidal dendrites and somata (DiFiglia et al., 1980; DiFiglia and Rafols, 1988). In addition to a high density of cell interactions, aspiny and pallidal neurons are postsynaptic to a variety of axon types. Golgi-EM studies have shown that at least 8 types of axons contact the dendrites of large aspiny neurons (DiFiglia and Carey, 1986); pallidal neurons receive a dense innervation of 3-6 types of terminals which differ in their relative distribution to somata and dendrites (see review by DiFiglia and Rafols, 1988). Moreover, pallidal neurons are heavily innervated by the axons of medium spiny cells which probably also contact medium and large caudate aspiny neurons via intrinsic axon collaterals.

In neostriatal aspiny and pallidal neurons, cell adhesion mediated by $\mathrm{NCAM}_{180,140}$ may be critical for maintaining the density and diversity of cell contacts or in regulating the surface area and/or location along the plasma membrane where various types of axons form contacts. There is evidence in the postnatal mouse cerebellum (Persohn and Schachner, 1987) that NCAM ${ }_{180}$, which has the longest cytoplasmic domain, becomes prevalent following the formation of stable cell contacts. Interestingly, in adult muscle cells NCAM is distributed heterogeneously in association with synaptic and perisynaptic sites (Covault and Sanes, 1986). In our study no correlation at the ultrastructural level could be found between patches of NCAM reaction product and the presence (or absence) of synaptic inputs. The use of serial section analysis and antibodies directed against $\mathrm{NCAM}_{140}$ or $\mathrm{NCAM}_{180}$, as well as those recognizing NCAM 120 , might clarify the relationship between NCAM patches and synaptic inputs.

\section{Dendritic spines and axons containing NCAM may be involved in formation of new contacts}

There is evidence from the study of denervated adult skeletal muscle that the distribution of NCAM is increased in both nerve and muscle cells during the period of muscle reinnervation (Covault and Sanes, 1985; Reiger et al., 1985). We suspect that a comparable situation may occur in the CNS and that the discrete localization of NCAM in a subpopulation of small, thin spines and in axons contacting small spines in the neostriatum represents a transient expression associated with newly stabilized contacts. There is evidence, described below, that neostriatal dendritic spines contribute to growth and plasticity in the normal and lesioned adult brain.

Ultrastructural immunohistochemical study of the normal rat caudate nucleus in our laboratory has shown that the growth associated protein, GAP-43, which is highly concentrated in the neostriatal neuropil (Benowitz et al., 1988), is localized to small axons which form asymmetric synapses with small dendritic spines (DiFiglia et al., 1989). Many of the GAP-43-positivc axons bear a striking similarity to the small NCAM-positive axon terminals found in this study to also contact small spines. Regenerative changes involving neostriatal spines have been observed in the lesioned brain. Following cortical deafferentation in mice there is a significant decrease and recovery in the density of dendritic spines in the caudate nucleus (Cheng et al., 1988). Intrastriatal injections of an excitotoxin, quinolinic acid, results in a significantly higher than expected density of small spines and axospinous synapses in regions of the caudate where there is a partial cell depletion (Roberts and DiFiglia, 1988). Finally, in the postmortem Huntington's disease brain (Graveland et al., 1985), where spiny neurons degenerate, the dendrites of remaining spiny neurons frequently elongate and proliferate spines. The extent to which cell adhesion mediated by NCAM may be involved in the regenerative response observed following neostriatal injury is currently being investigated in our laboratory.

\section{Future studies needed to clarify the role of NCAM}

In view of the known homophilic binding property of NCAM, we were surprised that so little NCAM immunoreactivity appeared along the plasma membranes of contiguous neuron-neuron or neuron-glial profiles. One possibility is that the unlabeled profiles apposing $\mathrm{NCAM}_{180,140}$-positive neurons contained $\mathrm{NCAM}_{120}$, the molecular subtype not identified by our antibody and previously found to be more prevalent in glial cells than in neurons (Noble et al., 1985; Bhat and Silberberg, 1986; He et al., 1986) or NgCAM which may interact with NCAM (Grumet et al., 1984). Other cell surface molecules which are present in neurons and glia (NILE, L1, Ng-CAM) and in the extracellular matrix (laminin, fibronectin, heparan sulfate proteoglycan) also facilitate adhesion (Sanes et al., 1986; Werz and Schachner, 1988). Their localization in relation to the cellular localization of NCAM 180,140 in the basal ganglia is unknown.

The expression of molecular subtypes of NCAM have been shown to vary with location and/or the functional state of the cell in other regions of the nervous system (Covault et al., 1986; Pollerberg et al., 1986; Persohn and Schachner, 1987). The different sites of NCAM localization observed in the basal ganglia (aspiny neurons, pallidal neurons, spines, small axons) may be associated with specific molecular subtypes which our monoclonal antibody cannot distinguish (NCAM1 80 and NCAM140). Future studies in the basal ganglia will be needed to clarify the relationship between neuronal localization and specific molecular forms of NCAM.

\section{References}

Beasley, L., and B. Stallcup (1987) The nerve growth factor-inducible large external (NILE) glycoprotein and neural cell adhesion molecule (N-CAM) have distinct patterns of expression in the developing rat central nervous system. J. Neurosci. 7: 708-715.

Benowitz, L. I., P. J. Apostolides, N. Perrone-Bizzozero, S. P. Finklestein, and H. Zwiers (1988) Anatomical distribution of growthassociated protein GAP-43/B-50 in the adult rat brain. J. Neurosci. 8: 339-352.

Bhat, S., and D. H. Silberberg (1986) Oligodendrocyte cell adhesion molecules are related to neural cell adhesion molecule (N-CAM). J. Neurosci. 6: 3348-3354.

Cheng, H.-W., Y. Anavi, T. H. Goshgarian, T. H. McNeill, and J. A. Rafols (1988) Loss and recovery of striatal dendritic spines following lesions in the cerebral cortex of adult and aged mice. Soc. Neurosci. Abstr. 14: 1292.

Chuong, C.-M., and G. M. Edelman (1984) Alterations in neural cell adhesion molecules during development of different regions of the nervous system. J. Neurosci. 4: 2354-2368.

Covault, J. (1989) Molecular biology of cell adhesion in neural development. In Molecular Biology, D. M. Glover and D. Hames, eds., pp. 143-200, IRL Press, Washington, D.C.

Covault, J., and J. R. Sanes (1985) NCAM accumulates in denervated and paralyzed skeletal muscles. Proc. Natl. Acad. Sci. USA 82: 45444548.

Covault, J., and J. R. Sanes (1986) Distribution of NCAM in synaptic and extrasynaptic portions of developing and adult skeletal muscle. J. Cell Biol. 102: 716-730.

Covault, J., J. P. Merlie, C. Goridis, and J. R. Sanes (1986) Molecular forms of N-CAM and its RNA in developing and denervated skeletal muscle. J. Cell Biol. 102: 731-739.

Cunningham, B. A., J. J. Hemperly, B. A. Murray, E. A. Prediger, R. 
Brackenbury, and G. M. Edelman (1987) Neural cell adhesion molecule: Structure, immunoglobulin-like domains, cell surface modulation, and alternative RNA splicing. Science 236: 799-806.

Daniloff, J. K., C. M. Chuong, G. Levi, and G. M. Edelman (1986) Differential distribution of cell adhesion molecules during histogenesis of the chick nervous system. J. Neurosci. 6: 739-758.

DiFiglia, M. (1987) Synaptic organization of cholinergic neurons in the monkey neostriatum. J. Comp. Neurol. 255: 245-258.

DiFiglia, M., and N. Aronin (1982) Ultrastructural features of immunoreactive somatostatin neurons in the rat caudate nucleus. $J$. Neurosci. 2: 1267-1274.

DiFiglia, M., and J. Carey (1986) Large neurons in the primate neostriatum, examined with the combined Golgi-electron microscopic method. J. Comp. Neurol. 244: 36-52.

DiFiglia, M., and J. A. Rafols (1988) Synaptic organization of the globus pallidus. J. Electron Microsc. Tech. 10: 247-263.

DiFiglia, M., T. Pasik, and P. Pasik (1980) Ultrastructure of Golgiimpregnated and gold-toned spiny and aspiny neurons in the monkey neostriatum. J. Neurocytol. 9: 471-492.

DiFiglia, M., R. C. Roberts, and L. I. Benowitz (1989) Ultrastructural localization of immunoreactive GAP-43 in the adult rat caudate nucleus. Soc. Neurosci. Abstr. 15: 910.

Edelman, G. M. (1983) Cell adhesion molecules. Science 219:450457.

Ferrante, R. J., N. W. Kowall, M. F. Beal, E. P. Richardson, E. D. Bird, and J. B. Martin (1985) Selective sparing of a class of striatal neurons in Huntington's disease. Science 230: 561-563.

Gennarini, G., G. Rougon, H. Deagostini-Bazin, M. Hirn, and C. Goridis (1984) Studies on the transmembrane disposition of the neural cell adhesion molecule N-CAM. Eur. J. Biochem. 142: 57-64.

Graveland, G. A., R. S. Williams, and M. DiFiglia (1985) Evidence for degenerative and regenerative changes in neostriatal spiny neurons in Huntington's disease. Science 227: 770-773.

Grumet, M., S. Hoffman, D.-M. Chuong, and G. M. Edelman (1984) Polypeptide components and binding functions of neuron-glia cell adhesion molecules. Proc. Natl. Acad. Sci. USA 81: 7989-7993.

He, H.-T., J. Barbet, J.-C. Chaix, and C. Goridis (1986) Phosphatidylinositol is involved in the membrane attachment of NCAM-120, the smallest component of the neural cell adhesion molecule. EMBO J. 5: 2489-2494.

Keilhauer, G., A. Faissner, and M. Schachner (1985) Differential inhibition of neurone-neurone, neurone-astrocyte and astrocyte-astrocyte adhesion by L1, L2 and N-CAM antibodies. Nature 316: 728730.

Kohler, G., and C. Milstein (1976) Continuous cultures of fused cells secreting antibody of predefined specificity. Nature 256: 495-497.

Noble, M., M. Albrechtsen, C. Moller, J. Lyles, E. Bock, C. Goridis, M. Watanabe, and U. Rutishauser (1985) Glial cells express N-CAM/ D2-CAM-like polypeptides in vitro. Nature $316: 725-728$.
Persohn, E., and M. Schachner (1987) Immunoelectron microscopic localization of the neural cell adhesion molecules L1 and N-CAM during postnatal development of the mouse cerebellum. J. Cell Biol. 105: 569-576.

Phelps, P. E., C. R. Houser, and J. E. Vaughn (1985) Immunocytochemical localization of choline acetyltransferase within the rat neostriatum: A correlated light and electron microscopic study of cholinergic neurons and synapses. J. Comp. Neurol. 238: 286-307.

Pollerberg, G. E., M. Schachner, and J. Davoust (1986) Differentiation state-dependent surface mobilities of two forms of the neural cell adhesion molecule. Nature 324: 462-465.

Rieger, F., M. Grumet, and G. M. Edelman (1985) N-CAM at the vertebrate neuromuscular junction. J. Cell Biol. 101: 285-293.

Roberts, R. C., and M. DiFiglia (1988) Ultrastructural changes in caudate neuropil following quinolinic acid lesions. Soc. Neurosci. Abstr. 14: 1025.

Rutishauser, U., A. Acheson, A. K. Hall, D. M. Mann, and J. Sunshine (1988) The neural cell adhesion molecule (NCAM) as a regulator of cell-cell interactions. Science 240: 53-57.

Sanes, J. R., M. Schachner, and J. Covault (1986) Expression of several adhesive macromolecules (NCAM, L1, J1, NILE, Uvomorulin, laminin, fibronectin, and a heparan sulfate proteoglycan) in embryonic, adult, and denervated adult skeletal muscle. J. Cell Biol. 102: 420431.

Silver, J., and U. Rutishauser (1984) Guidance of optic axons in vivo by a preformed adhesive pathway on neuroepithelial endfeet. Dev. Biol. 106: 485-499.

Thanos, S., F. Bonhoeffer, and U. Rutishauser (1984) Fiber-fiber interaction and tectal cues influence the development of the chicken retinotectal projection. Proc. Natl. Acad. Sci. USA 81: 1906-1910.

Thiery, J.-P., J.-L. Duband, U. Rutishauser, and G. M. Edelman (1982) Cell adhesion molecules in early chicken embryogenesis. Proc. Natl. Acad. Sci. USA 79: 6737-6741.

Tosney, K. W., M. Watanabe, L. Landmesser, and U. Rutishauser (1986) The distribution of NCAM in the chick hindlimb during axon outgrowth and synaptogenesis. Dev. Biol. 114: 437-452.

Towbin, H., T. Staehlin, and J. Gordon (1979) Electrophoretic transfer of proteins from polyacrylamide gels to nitrocellulose sheets. Procedure and some applications. Proc. Natl. Acad. Sci. USA 76: 43504354.

van den Pol, A. N., U. diPorzio, and U. Rutishauser (1986) Growth cone localization of neural cell adhesion molecule on central nervous system neurons in vitro. J. Cell Biol. 102: 2281-2294.

Werz, W., and M. Schachner (1988) Adhesion of neural cells to extracellular matrix constituents. Involvement of glycosaminoglycans and cell adhesion molecules. Dev. Brain Res. 43: 225-234. 